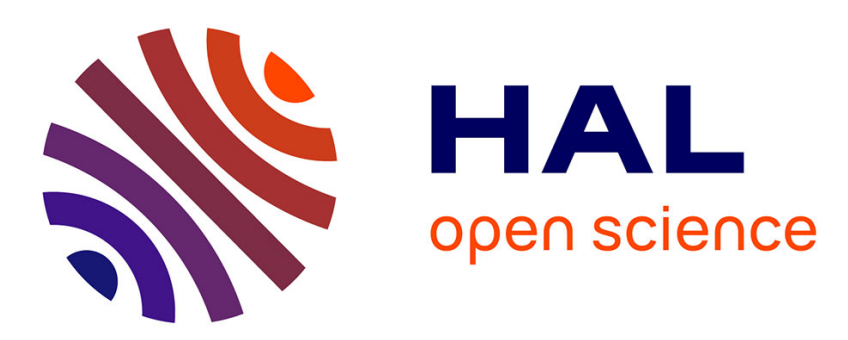

\title{
A Game at Chess de Thomas Middleton (1624): Echec et mat au théâtre du Globe
}

Antoine Ertle

\section{To cite this version:}

Antoine Ertle. A Game at Chess de Thomas Middleton (1624): Echec et mat au théâtre du Globe. Ligeia, dossiers sur l'art, 2019. hal-02137860

\section{HAL Id: hal-02137860 \\ https://hal.science/hal-02137860}

Submitted on 23 May 2019

HAL is a multi-disciplinary open access archive for the deposit and dissemination of scientific research documents, whether they are published or not. The documents may come from teaching and research institutions in France or abroad, or from public or private research centers.
L'archive ouverte pluridisciplinaire HAL, est destinée au dépôt et à la diffusion de documents scientifiques de niveau recherche, publiés ou non, émanant des établissements d'enseignement et de recherche français ou étrangers, des laboratoires publics ou privés. 


\title{
A GAME AT CHESS DE THOMAS MIDDLETON (1624) : ECHEC ET \\ MAT AU THEATRE DU GLOBE
}

\author{
Antoine Ertlé
}

Thomas Middleton n'est plus aujourd'hui le dramaturge de second rang dont T. S. Eliot disait au début du $\mathrm{XX}^{\text {ème }}$ siècle qu'il était «simplement un grand chroniqueur $^{1} \gg$. Les presses universitaires d'Oxford ont publié en 2007 ses œuvres complètes, accompagnées d'un volume critique particulièrement riche ${ }^{2}$. De nombreuses études lui sont consacrées en particulier depuis la fin des années 1950, et certaines de ses pièces ont désormais leur place dans les anthologies ou dans les répertoires aux côtés des œuvres de Marlowe, Chapman, Jonson, Massinger ou Webster et de celles de Shakespeare, figure tutélaire de ce théâtre élisabéthain et jacobéen. Des City Comedies comme A Chaste Maid in Cheapside, Michaelmas Term ou A Trick to Catch the Old One, des tragédies comme The Changeling, Women Beware Women ou The Revenger's Tragedy sont saluées par la critique comme autant d'œuvres majeures de la période.

Middleton voit sa carrière de dramaturge atteindre son apogée en 1624 avec $A$ Game at Chess (La Partie d'échecs ${ }^{3}$ ), une comédie satirique en forme de jeu d'échecs qui semble devoir échapper à toute classification. C'est en effet une pièce unique, et à bien des égards. Pour la première fois dans l'histoire de ce théâtre commercial né au siècle précédent, neuf représentations consécutives de la même pièce sont données sur la scène du Globe entre le 5 et le 14 août 1624. La foule se presse, les files d'attente s'allongent, les recettes dépassent tous les espoirs de la compagnie ${ }^{4}$. Ce

Eliot, T. S. Elizabethan Essays, Londres, Faber, 1934, p. 99.

2 G. Taylor et J. Lavagnino (dir.), Thomas Middleton : The Collected Works ; J. Lavagnino et G. Taylor (dir.), Thomas Middleton and Early Modern Textual Culture : A Companion to the Collected Works, OUP, Oxford, 2007.

3 Voir Thomas Middleton, A Game at Chess/ Une Partie d'échecs : édition bilingue établie par Antoine Ertlé, Paris: Epistémè, Université de la Sorbonne Nouvelle - Paris 3, 2004. (http://www.etudesepisteme.org/ee/articles.php?lng=fr\&pg=54).

4 Voir par exemple le témoignage de Don Carlos Coloma, ambassadeur d'Espagne, dans une lettre du 20 août adressée au comte-duc d'Olivares; de John Woolley, dans une lettre du 11 août adressée à William Trumbull, ou de 
succès remarquable suscite les réactions de nombreux observateurs, déroutés par l'ampleur de l'engouement et l'audace du propos. Car le succès de cette pièce est bien apparemment un succès de scandale. Les premiers témoignages des spectateurs d'alors identifient clairement la teneur véritable de la Partie d'échecs : c'est avant tout une pièce politique, dont les personnages ne sont pas les simples pions noirs et blancs d'une partie d'échecs, mais les acteurs de la scène politique de 1624, tous facilement identifiés par les spectateurs, et en premier lieu un certain Gondomar ${ }^{5}$, ancien ambassadeur d'Espagne à la cour d'Angleterre, véritable star de la pièce. D'autres figures politiques de l'époque sont représentées de manière très directe, au risque d'attirer les foudres du censeur, et le succès de la pièce s'explique ainsi en partie, sans que celle-ci puisse être réellement qualifiée d'œuvre de propagande, par son appartenance à un courant antiespagnol et anticatholique particulièrement prolifique à ce moment charnière de la vie politique anglaise.

Mais la forme et l'essence de cette Partie d'échecs la distinguent nettement des autres pièces ou pamphlets de l'époque. En effet, si les échecs sont bien connus du public élisabéthain et jacobéen, s'ils ont souvent été utilisés par les auteurs des Moralités, des pièces sur les trois États des périodes médiévales et Tudor, ou plus récemment sur les scènes du Globe, du Swan ou du Red Bull, ils donnent ici sa forme, sa couleur et son rythme à la pièce toute entière et deviennent le parfait véhicule du message complexe d'un spectacle étonnant, l'allégorie en forme de jeu d'échecs permettant à Middleton de mettre en scène des personnages et des événements contemporains de premier ordre.

Les personnages principaux incarnés par les Comédiens du Roi dans cette comédie qui attire une foule considérable, («toutes sortes de gens, vieux et jeunes, riches et pauvres, maitres et domestiques, papistes et puritains, sages, etc., hommes

John Holles dans une lettre du 11 août adressée au comte de Somerset, qui font tous état d'une foule de plus de trois mille spectateurs à chacune des représentations. (Cités par Howard-Hill, 1993, p. 194, 198 et 199 respectivement).

5 Diego Sarmiento de Acuña, comte de Gondomar, ambassadeur d'Espagne à Londres d'août 1613 à juillet 1618, puis de mars 1620 à avril 1622. C'est le Cavalier Noir de la Partie d'échecs. 
d'église et hommes d'état $\left.{ }^{6} »\right)$ sont les pièces anonymes d'une partie d'échecs, mais il est très facile de leur attribuer une identité ${ }^{7}$. Les Blancs sont les Anglais : parmi eux le Roi Jacques I $^{\text {er }}$ Stuart, la Reine, le Prince Charles, le Duc de Buckingham, et différents acteurs aux rôles moins importants, qui représentent pour certains tel ambassadeur à Madrid, pour d'autres tel archevêque ou homme d'État. Les Noirs sont les Espagnols : le Roi Philippe IV et sa Reine, son valido ou favori le Duc d'Olivares, le diplomate Gondomar et des Jésuites figurés par des pions. L'archevêque de Spalato, Marc Antonio de Dominis est un personnage particulièrement important, affublé du titre de Gros Fou, qui appartient d'abord à un camp puis à l'autre. Le Roi Noir convoite la Reine Blanche, le Cavalier Noir tente de persuader le Cavalier Blanc et son Duc de passer de l'autre côté, les Pions Noirs font tout leur possible pour s'emparer du Pion de la Reine Blanche, mais ce sont les Blancs qui l'emportent et jettent tout le camp Noir dans le même sac à la fin de la partie. La seule victoire des Noirs est d'avoir réussi à entraîner le Gros Fou dans leur chute.

Pour tous les spectateurs, il s'agit là d'une mise en scène d'événements bien connus, que l'on identifie plus ou moins aisément dans tel ou tel développement de l'intrigue : les longues négociations de mariage entre l'Angleterre et l'Espagne qui, quelques mois auparavant, ont fait planer la menace d'une restauration officielle $d u$ catholicisme, voire d'une conversion du Prince Charles, héritier du royaume; le voyage à Madrid de Charles et Buckingham dans le cadre de ces négociations; la perte du Palatinat au détriment de l'Électeur Frédéric et de son épouse Élisabeth, sœur du Prince Charles; les machinations de Gondomar à Londres visant à promouvoir la cause des Catholiques et à faciliter l'infiltration des Jésuites à la Cour et dans tout le royaume. La pièce a donc manifestement pour sujet les questions qui préoccupent à la fois le peuple anglais et les plus hautes sphères du pouvoir en ce mois d'août 1624, et pour personnages les plus hauts dirigeants de deux des

\footnotetext{
6 Lettre de John Chamberlain à Sir Dudley Carleton, ambassadeur à La Haye, 21 août 1624, Howard-Hill, Game, 1993, op. cit, p. 205. Ma traduction.

7 Voir tableau en annexe.
} 
principales nations d'Europe. C'est d'ailleurs à ce titre qu'elle est finalement interdite, en vertu d'une ancienne sanction rappelée par le Secrétaire d'État Edward Conway dans une lettre au Privy Council, 'a commaundment and restraint given against the representinge of anie moderne Christian Kings ${ }^{8}$, sur la scène d'un théâtre, confirmant une proclamation du 16 mai 1599 interdisant les pièces abordant les questions ouvertement religieuse ou politiques ${ }^{9}$.

Quant à l'auteur de cette pièce, ce n'est pas un inconnu. Il s'agit de Thomas Middleton, City Chronologer ${ }^{10}$ depuis 1620 , et auteur de nombreuses et célèbres comédies et tragédies. Sa pièce More Dissemblers Besides Women a été jouée à la Cour le 6 janvier 1624, et les divertissements qu'il écrit régulièrement pour le Lord Mayor de la ville de Londres lui valent la bienveillance d'un groupe d'hommes influents, proches des milieux parlementaires puritains et des nantis de la Cité.

Trois intrigues sont en fait habilement imbriquées au sein d'une trame principale constituée par la partie d'échecs. La première s'articule autour du personnage du Pion de la Reine Blanche, incarnation de la foi protestante, objet de la convoitise et de la concupiscence du camp Noir et en particulier des Pions ; le Cavalier Noir, double du détesté comte de Gondomar ${ }^{11}$, diplomate rusé et fin négociateur, l'accable et la condamne avec cruauté lorsqu'elle accuse le camp Noir d'en vouloir à son âme autant qu'à son honneur. Cependant, les coupables sont confondus et le malheureux Pion conserve sa candeur et peut rejoindre dans une union chaste et spirituelle le Pion du Fou Blanc, son premier et unique amour, castré au préalable par un Pion du Cavalier Noir particulièrement vicieux.

La deuxième intrigue a pour personnage principal le Gros Fou, incarnation fidèle de Marco Antonio de Dominis, archevêque de Spalato (la ville de Split, en Dalmatie), qui, venu du camp Noir, occupe maintenant d'importantes fonctions chez

8

Allusion, selon Janet Clare, à une promesse consentie par les compagnies de théâtre à la suite des pièces écrites par G. Chapman sur le Duc de Biron. J. Clare, 'Art Made Tongue-Tied by Authority': Elizabethan and Jacobean Dramatic Censorship, Manchester, 1990, p. 215.

${ }^{9}$ Howard-Hill, 1991, p. 278.

${ }^{10}$ Chroniqueur officiel de la municipalité de Londres.

${ }^{11}$ Les acteurs vont jusqu'à utiliser des costumes et des accessoires ayant appartenu à leur modèle pour rendre leur représentation plus crédible. 
les Blancs. Le Cavalier Noir s'efforce de ridiculiser le Gros Fou et de le faire revenir du côté du camp Noir. Il y parvient sans peine et celui-ci, qui tente maladroitement de capturer la Reine Blanche pour prouver la sincérité de sa foi retrouvée, est la première pièce majeure perdue par le camp Noir.

La troisième intrigue est la tentative du Cavalier Noir de persuader le Cavalier Blanc-le Prince Charles - et son Duc (c'est-à-dire sa Tour, sur l'échiquier) Buckingham, favori de Jacques $I^{\mathrm{er}}$ - de passer de l'autre côté. Les Blancs feignent d'être séduits, dans une scène qui évoque le séjour historique de Charles et Buckingham à Madrid de mars à août 1623, mais forcent habilement les Noirs à dévoiler leur fourberie et à oublier leur méfiance, et la pièce se termine par un retentissant «échec à la découverte».

Les témoignages contemporains font tous état en premier lieu de la forme particulière qui encadre le fond politique de la pièce. John Holles fait une description précise au comte de Somerset : «La pièce entière est un échiquier, l'Angleterre est la maison blanche, l'Espagne la noire ${ }^{12}{ }^{\prime}$ et ajoute une intéressante allusion au Pion du Roi Blanc, «l'un des pions blancs, dissimulant un pourpoint noir, signifiant un cœur espagnol $^{13} »$, qui nous indique que les spectateurs perçoivent en premier lieu des pions, des pièces noires ou blanches, obéissant aux règles du jeu d'échecs, puis découvrent, intrigués et ravis, les identités dissimulées plus ou moins subtilement par l'allégorie, là un Jésuite, là une fille de l'Église d'Angleterre, tel Roi ou tel ambassadeur tandis que les pièces s'animent et s'interpellent ${ }^{14}$.

Ils sont d'autant plus facilement identifiés que le jeu d'échecs n'est pas en 1624 une curiosité qu'un dramaturge raffiné voudrait utiliser pour signifier quelque propos obscur. Certes, les personnages des city comedies de Middleton jouent plus souvent aux cartes ou aux dés dans les tavernes de Cheapside, mais le jeu d'échecs n'en est pas moins dans l'air du temps. Middleton lui-même l'a déjà utilisé un ou deux ans

\footnotetext{
${ }_{12}^{12}$ Howard-Hill, Game, op. cit., p. 199. Ma traduction.

${ }_{13}^{13}$ Howard-Hill, Game, op. cit., p. 199. Ma traduction.

14
}

Dans la mise en scène de Mark Lambirth présentée à Cambridge en 1973, les pièces se déplaçaient en deux temps : un premier mouvement très rigide et muet, obéissant aux règles du jeu (deux pas en avant, un pas de côté pour le Cavalier), puis, en deuxième mouvement moins figé qui laissait place au dialogue. 
auparavant ${ }^{15}$ dans Women Beware Women. La scène 2 de l'acte 2 , très souvent citée comme exemple particulièrement probant du génie de Middleton ${ }^{16}$, propose une utilisation du jeu d'échecs où l'on retrouve, dans un contexte et dans des proportions pourtant toutes différentes, les multiples potentialités offertes par la métaphore du «plus noble des jeux ${ }^{17}$ ». Livia, une riche Florentine, est bien décidée à offrir au Duc la jeune Bianca, qui vient d'épouser Léantio. Elle invite la mère de celui-ci à venir lui rendre visite en compagnie de sa bru. Guardiano, un ami de Livia, fait visiter la maison à Bianca tandis que Livia et son invitée jouent aux échecs sur la scène principale. Commence alors une double partie magistralement orchestrée à deux niveaux, littéralement, de signification : d'une part la partie d'échecs sur la scène principale, ponctuée d'explications tactiques, de captures, de manœuvres ; de l'autre la «prise » de Bianca, trompée par Guardiano, attrapée par le Duc, sur la scène supérieure, le balcon du théâtre. Rigoureuse superposition spatiale à laquelle correspond une stricte correspondance verbale entre les deux scènes, utilisant toutes deux la polysémie des mots anglais game (jeu et proie, cour), take (prendre, prise), piece (pièce, partie du corps) ou stroke (manœuvre, saisie) et le double sens du titre de Duc, la Tour du jeu d'échecs étant appelée Duke en anglais au XVII ${ }^{\text {ème }}$ siècle.

Middleton n'en est donc pas à sa première Partie d'échecs. Cependant, la reprise de la métaphore échiquéenne dans la Partie d'échecs est bien plus qu'une simple adaptation d'un artifice à de nouvelles circonstances.

Dans Women Beware Women, l'utilisation brillante du jeu d'échecs comme métaphore de la conquête amoureuse n'est qu'un procédé ponctuel, une trouvaille qui permet à Middleton de rendre compte de la brutalité et de l'inexorabilité de la capture de Bianca. Livia et la mère de Léantio jouent effectivement aux échecs, tandis que le duc et Bianca ne font que jouer leur rôle, sans faire pour autant partie, comme les personnages de la Partie d'échecs, d'une allégorie. Dans la Partie d'échecs, les

${ }^{15}$ Dorothy Farr situe la pièce après The Changeling (1622), soit immédiatement avant la Partie d'échecs.

${ }^{16}$ Dans sa critique de la mise en scène de Marianne Elliott présentée au théâtre Olivier de Londres en 2010, Susannah Clapp décrit la scène comme une « wonderful, pivotal scene in which Tilly Tremayne's well-judged, shrewd widow takes on Harriet Walter's glittering bawd at chess ». https://www.theguardian.com/stage/2010/may/02/womenbeware-middleton-bingo-bond

\footnotetext{
${ }^{17}$ A Ertlé, éd., Une Partie d'échecs, Induction, 42.
} 
personnages sont à la fois les pièces d'une allégorie permanente et totale, annoncée clairement par le Prologue et l'Induction, et les joueurs conscients du jeu qu'on leur impose. Ils font ainsi de nombreux commentaires que l'on pourrait qualifier d'internes à l'allégorie, dont les effets sont apparemment contradictoires : ils nuisent d'une part à la cohérence de l'allégorie (les pièces cessent d'être de simples pièces pour devenir des personnages de théâtre en train de jouer aux échecs), mais en rompent par là même la potentielle monotonie et le caractère unidimensionnel : les pièces ne sont plus simplement des pièces, mais aussi des joueurs, non plus simplement manipulés, mais manipulateurs, créant ainsi une nouvelle dimension, une nouvelle profondeur, qui permet de comparer la Partie d'échecs toute entière à l'éblouissante mise en abyme du balcon de Women Beware Women. Cette double dimension de la Partie d'échecs est d'autant plus frappante qu'elle n'apparaît qu'après une longue introduction où la cohérence de l'allégorie reste entière. Les pions de l'acte 1 sont bien les pions d'un jeu d'échecs, et s'identifient comme tel : «C'était donc le Pion du Fou Blanc» (1.157), «Le Pion du Cavalier Noir, que j'exècre pour l'éternité » (1.160), mais utilisent «Monsieur», «Madame », «Ma fille» ou «plus noble des vierges » dans leurs conversations, montrant ainsi leur détachement par rapport à l'allégorie dont ils font partie, leur inconscience de cette appartenance. Tous les termes renvoyant aux échecs dans ce premier acte de la pièce, les noms des personnages, sont en quelque sorte employés au premier degré : des hommes et des femmes présentent une pièce qui est une partie d'échecs, et il est donc normal qu'ils soient blancs ou noirs, que leur nom soit le nom d'une pièce. Les choses se compliquent avec l'arrivée du Cavalier Noir qui s'entretient avec son Pion. Celui-ci semble alors ouvrir la porte de l'autre dimension évoquée plus haut. $\mathrm{Au}$ Cavalier qui l'interroge sur son « jouet», le Pion de la Reine Blanche, il répond :

Mon jouet dites-vous? C'est un pion de valeur,

Chez les pions du camp Blanc elle vient en second rang,

Et je la serre de près pour ouvrir la partie. 
ce à quoi son maître rétorque : «Pour serrer de près, il s'y entend ; je connais son jeu depuis bien longtemps » (1.290). Ces deux personnages qui parlent d'échecs, qui commentent en fins tacticiens le début de partie que nous venons de suivre, inaugurent alors une série d'incursions en quelque sorte hétérodiégétiques, qui nous rappellent à intervalles réguliers que ces personnages noirs et blancs évoluant sur scène dans une allégorie savent parfois s'en échapper et prendre du relief. Le Cavalier Noir est bien souvent l'auteur de ces interventions :

Aucun coup de ce jeu ne pourra t'épargner.

Le Fou, moi-même, ce pion ou bien cet autre

Finirons par te prendre, quelle que soit ta défense.

(2.2.182-184)

« Je jouerai donc ainsi »(2.2.189) annonce-t-il fièrement avant de s'attaquer au Pion de la Reine Blanche. Il offre plus tard ses services à son roi :

Sire, je peux capturer un pion sans plus attendre.

Je sais par où le prendre.

(3.1.251-252)

« à notre coup de maître » (3.1.19), déclare-t-il en aparté avant de s'en prendre au Gros Fou, qui observe, prudent : «Il me faut jouer prudemment » (3.1.23),

Je ne connais que trop les coups du Cavalier ;

S'il me saute dessus, qu'adviendra-t-il de moi ?

(3.1.29-30) 
Le Pion du Fou Noir est lui aussi un maître du jeu et déclare, irrité, au Pion du Cavalier Noir: «Eh bien, que voulez-vous ?/J'ai un coup à jouer. (4.1.18-19), comme si ce prétentieux avait mieux à faire que de jouer les Pions dans la Partie de Middleton. Quand il aperçoit le Pion de la Reine Blanche, il déclare :

Voici la pièce convoitée. Jouons comme un grand maître,

Et feignons de ne pas la voir.

(4.1.34-35)

avant de s'interroger sur l'efficacité de son attaque : «Que jouer à présent ? » (4.1.134).

Les références internes au jeu d'échecs se font plus fréquentes à la fin de la pièce, où les personnages se dévoilent et revêtent ainsi tous cette autre dimension à peine entrevue jusque-là. Les Blancs apparaissent alors eux aussi, mais bien tardivement, comme des maîtres du jeu :

Eh bien continuez!

La partie est à nous. Nous te mettons, ô Roi,

En échec à la découverte, le plus noble des mats.

(5.3.167-169)

déclare le Cavalier Blanc, après avoir longtemps caché son jeu, tout juste annoncé à demi-mot au début de la pièce :

Ils peuvent toujours prendre les pires résolutions,

Si mon juste projet parvient jusqu'à sa fin.

(2.2.244-245)

Cette utilisation particulièrement riche de l'allégorie échiquéenne est sans doute la grande nouveauté de la pièce de Thomas Middleton. On y assiste en effet à une 
exploitation systématique des différentes significations possibles d'une métaphore courante mais souvent limitée à un seul niveau de sens.

George Chapman, par exemple, utilise le jeu d'échecs dans une scène de Bussy d'Ambois (1604 ou 1607) qui rappelle la scène 2 de l'acte 2 de Women Beware Women. Le passage commence par la didascalie suivante: 'Table, chessboard, and tapers, HENRY, GUISE [playing chess] ${ }^{18}$, et l'on assiste alors à une partie d'échecs entre Guise et le roi de France, métaphore de la rivalité, de l'affrontement, à laquelle correspond une autre partie, une autre tentative de conquête ou de suprématie, à savoir la cour que fait ouvertement Bussy d'Amboise à la duchesse de Guise, et qui fait dire au courtisan Barrisor :

Qui est donc ce nouveau venu, qui ose mater

Le Guise de la sorte?

(1.2.108-109)

Le double sens de mate introduit l'autre signification métaphorique du jeu d'échecs, c'est-à-dire la conquête amoureuse. On retrouve dans la scène de Chapman le même jeu sur les titres, qui sont aussi le nom des pièces du jeu, que dans Women Beware Women : «Pardieu, c'est Amboise ; le Duc l'aura pris, par ma vie, pour l'un de ces Chevaliers nouvellement apparus » dit l'Anou (1.2.110-111), rendant ainsi explicite, comme l'avait fait Livia en parlant de son Duc, le lien entre les deux «parties », et glissant au passage une intéressante référence aux nouveaux chevaliers qui apparaissent dans les premières années du règne de Jacques I ${ }^{\mathrm{er19}}$.

L'allégorie du jeu d'échecs propose à la fois une métaphore du statut et de l'ordre social (la valeur, la place et le rayon d'action des pièces) et de la politique (les règles, la stratégie) que l'on retrouve dans l'intrigue principale de la pièce, et une

\footnotetext{
${ }^{18}$ George Chapman, Bussy d'Ambois, acte 1, scène 2. Les citations font référence à l'édition de Nicholas Brooke (Londres, 1964), pp. 16-28. Ma traduction.

${ }^{19}$ En deux mois, il crée plus de nouveaux titres qu’Élisabeth durant les dix années précédentes.
} 
métaphore de la séduction et de la conquête amoureuse, plus ou moins courtoise, que l'on retrouve dans l'intrigue dite secondaire.

Les connotations politiques et sociales des échecs sont évidentes depuis la naissance du jeu. Dans le Liber de Moribus du moine dominicain Jacques de Cessoles traduit en anglais par William Caxton en 1474, le jeu est présenté comme l'invention d'un philosophe «pour corriger et censurer le $\operatorname{Roi}^{20} »$. Gustavus Selenus qualifie le jeu de König-Spiel ${ }^{21}$. Le roi Jacques I ${ }^{\text {er }}$ lui-même n'hésite pas à utiliser la métaphore du jeu d'échecs, qu'il ne tient pourtant pas en très haute estime (« Je le juge trop frivole car c'est une folie qui se veut trop sage et philosophique $\left.{ }^{22} »\right)$. Dans un discours au Parlement, il se sert de la métaphore pour exprimer, avec une grande habileté, sa conception absolutiste du pouvoir royal : «Les Rois ont le pouvoir d'exalter ce qui est inférieur et d'abaisser ce qui est supérieur, et d'agir avec leurs sujets comme avec les pièces d'un jeu d'échecs ; un pion pour prendre un Fou ou un Cavalier, et pour promouvoir ou rabaisser quiconque parmi leurs sujets ${ }^{23}$.» Curieusement, le Parlement utilise les mêmes armes rhétoriques, et Sir Benjamin Rudyerd, qui d'après John Chamberlain assiste à une représentation de la Partie d'échecs ${ }^{24}$, parle du Palatinat lors des débats sur le mariage de Charles et de l'Infante, comme d'une « pièce perdue ${ }^{25} »$.

L'allégorie d'une partie d'échecs où se rencontrent rois et parlementaires va réapparaître en Angleterre à un moment de son histoire où l'affrontement entre les adversaires s'apprête à prendre une tournure dramatique. Le pamphlet attribué à William Cartwright de 1642 intitulé The Game at Chesse est un exemple particulièrement abouti d'utilisation politique de la métaphore échiquéenne, dans le contexte de la Guerre Civile. Les Blancs représentent l'armée parlementaire, les Noirs, l'armée du roi. L'auteur justifie ainsi le choix d'un tel artifice: «Mon

\footnotetext{
${ }^{20}$ Jacques de Cessoles, Liber de Moribus («Livre des mœurs des hommes et des devoirs des nobles, au travers du jeu d'échecs », c. 1315) traduction de William Caxton, cité par S. Chakravorty, Society and Politics, p. 166. Ma traduction.

${ }^{21}$ Gustavus Selenus, Das Schach - oder König-Spiel, Leipzig, 1616.

${ }^{22}$ Cité par Paul Yachnin, 1982, p. 317. Ma traduction.

${ }^{23}$ Ibid. Ma traduction.

${ }^{24}$ Howard-Hill, Game, op. cit., p. 205.

${ }^{25}$ Howard-Hill, 1985, p. 6.
} 
intention étant seulement d'exprimer les affaires de ce Royaume, qui peuvent pertinemment être décrites allégoriquement sous la forme d'une bataille d'échecs » (p. 3), et apporte une précision qui n'est pas sans rapport avec la pièce de Middleton : «Le [jeu] d'échecs est uniquement dirigé par la force de l'esprit et du jugement : les Espagnols, dont la gravité du port est célèbre dans toutes les nations, le portent en si haute estime qu'ils déclarent gentilhomme tout étranger qui sait jouer aux échecs » (p. 3-4). On comprend mieux à travers cette référence au statut prestigieux dont jouissent les échecs en Espagne, l'indignation de l'ambassadeur Coloma face à la satire de Middleton, qui n'hésite pas à désacraliser ce que Ruy López, Alfonso Cerón ou Gioachino Greco élevé aux XVI ${ }^{\text {ème }}$ et XVII ${ }^{\text {ème }}$ siècles au rang de grand $\operatorname{art}^{26}$. La Guerre Civile se prête particulièrement bien à ce traitement allégorique par le biais des échecs. Par un retournement ironique saisissant, l'histoire a voulu que la guerre se termine par un échec et mat loin d'être allégorique. Charles $\mathrm{I}^{\mathrm{er}}$ Stuart, le Cavalier Blanc de Middleton, promu Roi Noir dans le pamphlet de Cartwright, sera décapité en 1649 .

La dimension politique de la métaphore échiquéenne, que nous venons d'aborder, est sans doute la plus exploitée par les dramaturges, les poètes ou les philosophes. Elle se rencontre aujourd'hui fréquemment dans la presse, particulièrement friande de métaphores et d'allégories, pour laquelle, après l'apport de nouvelles connotations dues à la domination du jeu par les Soviétiques durant une bonne partie du XX $X^{\text {ème }}$ siècle et de la Guerre froide, elle reste, comme en 1624, un excellent moyen de figurer l'affrontement, les conflits d'intérêts, les combats politiques et les guerres.

A l'instar du Roi et du Parlement, l'Église n'hésite pas, malgré une attitude longtemps hostile envers le jeu, à utiliser la métaphore de la partie d'échecs. Dans un sermon de 1614 prononcé par le célèbre prêcheur puritain Robert Harris le jour des funérailles de son protecteur, le parlementaire puritain d'Oxford Sir Anthony Cope, on trouve la référence suivante : «Lors d'une partie d'échecs, certains sont Rois,

\footnotetext{
${ }^{26}$ Voir J. M. Péchiné, Les Échecs, op. cit., p. 51-52.
} 
certains sont Fous, etc. mais après un moment, tous se retrouvent dans le même $\operatorname{sac}^{27}$. $\gg$ La métaphore renforce ici le message du pasteur sur l'égalité devant la mort et sur son caractère inéluctable. On touche là à l'interprétation la plus intéressante de cet aspect politique de la métaphore des échecs. A la fin de la partie, toutes les pièces sont dans le même sac. Ce sac, sur la scène, est le strict équivalent de la bouche de l'Enfer telle qu'on la devine à la fin du Doctor Faustus de Christopher Marlowe ${ }^{28}$. Il y a nivellement par l'allégorie, effacement de la hiérarchie par la chute finale de toutes les pièces du jeu. Pour Paul Yachnin, Middleton, comme nombre de ses prédécesseurs, exploite ici un mode homilétique conventionnel afin d'exprimer un message politique. La remarque du Cavalier Noir : «Le sac est assez grand, nous y logerons tous !» (3.1.311) n'est pas sans évoquer le message du sermon de Robert Harris.

Mais ce nivellement opéré par l'allégorie introduit une certaine ambiguïté. Malgré leurs noms différents, la place qu'ils occupent et les marges de manœuvre qui leur sont imparties, tous les personnages, toutes les pièces obéissent aux mêmes règles dans un même espace. Cet égalitarisme est illustré par l'histoire des échecs, au cours de laquelle on assiste à un équilibrage progressif des forces des différentes pièces, afin de rendre le jeu plus difficile et donc plus captivant. Le Fou dispose de toute la diagonale, le Pion peut au départ enjamber deux cases et se changer en Reine, qui dispose, elle, de toute latitude pour se rendre où elle veut ${ }^{29}$. Ainsi, malgré ses protestations ( « Le Fou doit avoir de la place, et encore de la place,/Et bien assez de place pour s'étendre à son aise », 5.3.192-193), le Gros Fou n'a pas droit à un traitement de faveur dans le sac où disparaissent toutes notions de respect ou de préséance («Je répugne à bouger pour un roi quel qu'il soit», 5.3.201; « Ma foi, [...] une Reine peut attirer un Fou », 5.3.203), et c'est la démesure de son orgueil qui lui fait nier l'évidence :

\footnotetext{
${ }^{27}$ Cité par Jeanne Shami, 'Thomas Middleton's A Game at Chess: A Sermon Analogue', p. 368. Ma traduction.

28 'And there behold: the bag, like hell-mouth, opens/To take her due...', A Game at Chess, 5.3., pp. 179-180 ; 'Contention in the pit! Is hell divided?', 5.3., p. 197.

${ }^{29}$ Voir J. M. Péchiné, Les Échecs, op. cit., pp. 33-55, sur la fixation des règles modernes du jeu d'échecs.
} 
Mettez-y tout ce que vous voudrez,

Le Fou sera toujours la pièce principale,

Malgré le Roi, la Reine ou le politicien.

(5.3.213-215)

Le nivellement défini plus haut est donc restreint à certains personnages, même s'il opère effectivement à l'intérieur de chacun des deux camps, autre adéquation entre calvinisme et échecs : il y a des différences de fonctions entre les pièces ou les hommes, mais la même nature fondamentale, la même égalité devant Dieu ou devant les règles du jeu. C'est pourquoi le Pion du Fou Noir se permet de répondre à son roi qui lui demande de s'emparer de la Reine Blanche :

Sire, lorsque mon désir sera enfin guéri

De cette inflammation qui brûle encore en moi, J'aurai tout le loisir d'examiner le vôtre.

$(2.1 .25-27)$

Conséquence curieuse de l'ambiguïté qui sous-tend la Partie d'échecs, la disparition du contraste pourtant apparemment si vif entre le blanc et le noir. Dans la scène 3 de l'acte 3 , où le Pion de la Reine Noire invoque le «parfait gentilhomme » dans son miroir magique, les Reines, la Blanche comme la Noire, sont impuissantes à le faire apparaître. Les pions facétieux de la scène précédente, s'ils usent et abusent des termes blancs et noirs, sont indifférenciés à la fin de leur joute, dilués dans la comédie :

Nous voilà pareils à trois mouches

Avec une paille dans le derrière.

$(3.2 .38-39)$ 
Le noir et le blanc des échecs, constamment juxtaposés, parfaitement symétriques, finissent par se confondre, pour faire de la pièce l'une de ces anamorphoses en vogue au XVI ${ }^{\text {ème }}$ et au XVII ${ }^{\text {ème }}$ siècles. Le contraste si net entre les deux couleurs est estompé par le va-et-vient incessant des pièces sur l'échiquier, à la manière d'un cercle bicolore, qui, par une illusion d'optique, devient uniformément gris quand on le fait tourner. C'est là un des effets les plus inattendus de cette allégorie en forme de jeu d'échecs : au lieu d'un manichéisme rassurant, elle nous offre un tableau changeant, où, dans un troublant clair-obscur, il est difficile de distinguer le Bien du Mal, le Blanc du Noir, le jeu de la réalité : «le passage incessant du blanc au noir, du sérieux à la comédie, nous aveugle et nous prive du réconfort moral de l'allégorie », écrit $\mathrm{S}$. Chakravorty ${ }^{30}$. L'alternance des couleurs et la confusion qu'elle entraîne, le principe de dualité, de dédoublement, de duplicité qui régit l'allégorie tout entière, font naître une ambiguïté diffuse d'un bout à l'autre de la Partie d'échecs.

Il est un sens de game, dont la polysémie est très souvent exploitée par la théâtre jacobéen, et par conséquent une interprétation de l'allégorie, qu'il reste à aborder pour compléter cette tentative de déchiffrage de la Partie d'échecs : celui de gibier, de proie, de victime de la joute amoureuse. Quand le Pion du Fou Noir dit en apercevant le Pion de la Reine Blanche : «Voici la pièce convoitée. Jouons comme un grand maître,/Et feignons de ne pas la voir. »(4.1.34-35), il veut, en utilisant le mot game, (yonder's my game) parler de son jeu, du coup qu'il va jouer dans la partie d'échecs, mais aussi de la proie qu'il s'apprête à chasser, et plus précisément de l'objet de sa concupiscence. Le Pion du Fou Noir déclare à sa victime qui croit venue l'heure du mariage : « Vous êtes toute enflammée par votre bonne affaire » (5.2.58),

Me reconnaissez-vous, luxure, iniquité,

Sainte prostitution, si prompte à vous marier,

\footnotetext{
${ }^{30}$ Society and Politics, op. cit., p. 173.
} 
Poussée par le désir, pour sauver la partie ?

$(5.2 .60-62)$

S'il y a là manifestement une dénonciation très brutale du mariage espagnol, de ce Game at Chess entre l'Angleterre et l'Espagne, il y a aussi une évocation de la couleur morale caractéristique du théâtre jacobéen, où l'amour est une commodité, la virginité un article de choix qu'il convient de bien négocier, le mariage une opération financière et l'étape obligée d'une ascension sociale, où le Mal a pour nom luxure, où la Faute, le blood's game du Pion de la Reine Noire (4.1.148), est toujours sexuelle. C'est ce game sexuel et sordide qui donne sa noirceur à la Partie d'échecs. Le Pion de la Reine Blanche ne devine pas les intentions de son pieux confesseur car elle joue à un autre jeu. Son monde est celui des échecs courtois des allégories médiévales. Elle est aimable, respectueuse, obéissante, et ne s'écarte jamais du chemin de la vertu $^{31}$, sans savoir qu'il la mène tout droit dans la gueule du loup (« Le destin est étrange : la voilà capturée, et par son pire ennemi. », 3.3.71).

Après avoir tenté de cerner la richesse de l'allégorie et les différentes interprétations qu'elle induit, il reste à définir les origines précises de l'allégorie de la Partie d'échecs, à identifier les multiples sources de la pièce, pour en faire ressortir les fonctions respectives.

Les critiques ont depuis longtemps inventorié les sources utilisées par Middleton pour faire de sa pièce une partie d'échecs ${ }^{32}$. H. J. R. Murray est le premier à signaler ${ }^{33}$ les possibles emprunts à une œuvre remarquable de la fin du $\mathrm{XV}^{\text {ème }}$ siècle, Le Songe d'amour de Poliphile ${ }^{34}$, monument inclassable de l'art complexe et raffiné des

${ }^{31}$ Voir en particulier 1.7-16, 90-95, 141-143, 182-184, 191-192 et 2.1.1-8, pp. 45-48.

${ }^{32}$ Voir en particulier J. R. Moore, «The Contemporary Significance of Middleton's A Game at Chess », in $P M L A, \mathrm{n}^{\circ}$ 50, 1935, pp. 761-768; David F. George, «Thomas Middleton's Sources: A Survey », in $N \& Q, \mathrm{n}^{\circ} 216$, 1971, p. 17-22 ; N. W. Bawcutt, « New Light on Middleton's Knowledge of Chess », in $N \& Q$, n ${ }^{\circ} 232,1987$, pp. 301302 ; et Paul Yachnin, «A Game at Chess and Chess Allegory », in SEL, n 22, 1982, pp. 317-330.

${ }^{33}$ H. J. R. Murray, A History of Chess, Oxford, 1913, p. 748.

${ }^{34}$ Terminé en 1465 et publié à Venise en 1499 par Aldo Manuzio, Hypnerotomachia Poliphili est attribué à Francisco Colonna ou, plus récemment, à Leon-Battista Alberti. Voir Emanuela Kretzulesco-Quaranta, Les jardins du songe, Paris, 1986 et Liane Lefaivre, Leon Battista Alberti's Hyptnerotomachia Poliphili, Boston, Mass., 1997. 
humanistes italiens du quattrocento. Dans ce roman polyglotte, le pèlerin Poliphile s'endort à l'orée d'une forêt et voit, dans son rêve, un ballet offert à la Reine, dansé par trente-deux acteurs. Il s'agit en fait d'une partie d'échecs, annoncée dès l'arrivée de Poliphile dans le palais, dont le dallage a l'aspect d'un échiquier. La description de la partie n'est pas sans évoquer l'Induction de la pièce de Thomas Middleton. Le jeu que l'Erreur va présenter à Saint Ignace n'est qu'un rêve, tout comme le songe de Poliphile ${ }^{35}$

Oh, je pourrais dormir dans l'ignorance

Jusqu'à la fin des temps, le sommeil est si doux !

Je rêvais à l'instant du jeu le plus brillant

Qu'il m'ait jamais été donné de contempler.

(Ind.38-41)

et la description des pièces sur le point de commencer la partie évoque souvent la majestueuse chorégraphie onirique de Poliphile. Toutefois, même si nous avons dans le Songe de Poliphile et dans l'Induction de la pièce de Middleton une partie d'échecs dansée qui n'est en fait qu'une vision onirique, l'influence directe de la première œuvre sur la seconde est difficile à établir de façon irréfutable. Le seul élément véritablement probant est la reprise par Middleton de l'expression « Custode de le Roche » pour désigner la Tour, mais rien n'indique que Middleton n'ait pas rencontré l'expression ailleurs ${ }^{36}$. Une traduction anglaise incomplète du Songe de Poliphile paraît en 1592, mais il semble que Middleton ait eu accès à l'œuvre par l'intermédiaire de Rabelais ${ }^{37}$. Pour Paul Yachnin, «il est presque certain que

${ }^{35}$ Ce procédé est à rapprocher du rituel du rêve, 'a special instance of playhouse games' au Moyen-Age, selon M. C. Bradbrook (English Dramatic Form: A History of its Development, Londres, 1965), qui projette d'emblée le spectateur dans un monde imaginaire, le transporte dans une autre dimension, à la manière des Prologues du théâtre élisabéthain. Voir à ce sujet les remarques de S. Chakravorty, Society and Politics, op. cit., p. 167.

${ }^{36}$ Rabelais utilise l'expression «Custodes de la Rocque » dans sa description du bal donné au Palais de la Quinte Essence. Rabelais, Le Cinquième Livre, in Les Cinq Livres, éd. Jean Céard, Gérard Defaux et Michel Simonin, Paris, 1994, p. 1409.

${ }^{37}$ Cette hypothèse est développée par Paul Yachnin, 1982. 
Middleton a lu Rabelais ${ }^{38} »$. Au chapitre XXIII du Cinquième Livre, Rabelais décrit un bal en forme de jeu d'échecs, donné dans le palais de la Quinte Essence devant Pantagruel et ses compagnons, bal qui s'inspire manifestement du Songe de Poliphile $^{39}$. Dans sa description des différents acteurs de la partie, Rabelais utilise le terme « Archiers » pour désigner les Fous. Dans la version italienne du ballet, ce sont des «Secrétaires ». L'archer est fréquemment représenté sur l'échiquier depuis les origines indiennes et persanes du jeu, mais Rabelais emprunte peut-être le sien à une autre allégorie, le Scacchia Ludus de Marco Girolamo Vida (1485-1556), poème latin écrit vers $1510^{40}$ et publié pour la première fois en 1525 . Une traduction anglaise (d'un G. B. non identifié) parait en 1597, accompagnée d'une version modernisée de la traduction de James Rowbothum dédiée à Sir Robert Dudley du Libro da imparare giocare a scachi de Damiano da Odenara, publiée en 1562 sous le titre The pleasaunt and vvittie playe of the cheasts. Plusieurs éléments semblent en effet rapprocher le ballet raconté par Pantagruel de l'épopée virgilienne du poète de Crémone. Scacchia Ludus n'est plus un ballet mais un combat aux proportions impressionnantes, offert aux Dieux cruels, empreint du souffle et de l'ampleur d'une terrible gigantomachie. Le Fou, donc, est «le jeune homme aimé de Mars, au remarquable arc d'ivoire ${ }^{41} »$. Dans la Partie d'échecs, le Fou n'est pas un archer, mais le Pion du Fou Blanc, amoureux du Pion de la Reine Blanche, offre ses services au Cavalier Blanc en des termes qui rappellent cette association :

Laissez-moi cet honneur, Monsieur.

Faites de moi cette flèche [flight], d'elle mon unique but.

(2.2.240-241)

\footnotetext{
${ }^{38}$ Paul Yachnin, 1982, p. 318.

${ }^{39}$ Rabelais cite le titre de l'ouvrage de Colonna (ou Alberti) au chapitre IX de La Vie Treshorrificque du Grand Gargantua, «Les couleurs et livrée de Gargantua ». Voir l'édition de Mireille Huchon et François Moreau, Bibliothèque de la Pléiade, Paris, 1994, pp. 28-30.

40 Voir Paul Yachnin, 1982, p. 319.

${ }^{41}$ Marco Girolamo Vida, Scacchia Ludus, in Musae Reduces, Anthologie de poésie latine européenne de la Renaissance, Leiden, 1975, tome II, p. 233, vers 595-596.
} 
où flight désigne une longue flèche utilisée pour atteindre une cible lointaine avec une grande précision ${ }^{42}$.

Un autre point commun entre l'œuvre de Rabelais et celle de Vida permet d'établir un lien plus convaincant entre la Partie d'échecs et les allégories française et italienne. Le Cavalier est clairement identifié à une pièce fourbe et particulièrement dangereuse. Dans Scacchia Ludus, sa redoutable efficacité est évoquée comme suit :

Alors s'exécute un nouveau coup, qui va infliger à Phébus une douleur plus grande et plus cruelle : Mercure presse son noir destrier de tous côtés et saute sur le terrain découvert. Le cheval ardent fait des bonds furieux; et il n'a de cesse qu'il n'ait menacé à la fois la reine et le roi en rejoignant la position souhaitée ; et pour mettre en échec l'une ou l'autre, il les accule avec ses armes noires ${ }^{43}$.

Pour leur part, les Chevaliers de Rabelais marchent et prenent en forme ligneare, passans un siege franc, encores qu'il fust occupé, ou des siens, ou des ennemis : et au second soy posans à dextre ou à senestre, en variation de couleur, qui est sault grandement dommageable à partie adverse, et de grande observation : car ils ne prenent jamais à face ouverte ${ }^{44}$.

Ce déplacement « en forme ligneare », c'est-à-dire en forme de potence ${ }^{45}$ - cette potence que le Cavalier Noir réserve au Gros Fou ( $\mathrm{A}$ mon tour de le pendre », 3.1.291) - ces sauts « grandement dommageable[s] », rappellent les sinistres talents du Cavalier de Middleton, redoutés par ses adversaires :

Je ne connais que trop les coups du Cavalier ;

S'il me saute dessus, qu'adviendra-t-il de moi ?

(3.1.29-30)

\footnotetext{
${ }^{42}$ Howard-Hill, Game, op. cit., p. 118.

${ }^{43}$ Marco Girolamo Vida, op. cit., vers 575-582.

${ }^{44}$ Rabelais, op. cit., p. 1411.

${ }^{45}$ Rabelais, op. cit., note p. 1410.
} 
En outre, les manœuvres du Chevalier Argenté sont parfois très proches de celles du Cavalier Noir :

Mais le Chevalier argenté pense chose plus importante, dissimulant son entreprinse, et quelquefois qu'il a peu prendre une Nymphe aurée, il la laisse et passe outre, et a tant faict qu'il s'est posé près ses ennemis ${ }^{46}$.

C'est un renard de la même espèce que celui qui déclare :

Je m'apprête à jouer de façon magistrale

Pour piéger le Cavalier Blanc, en le trompant

Pour l'attirer dans le camp Noir - d'autres suivront.

Que nous indiquent ces comparaisons qui font apparaître une incontestable parenté entre des œuvres telles que Le Songe de Poliphile, Scacchia Ludus, Le Cinquième Livre et la Partie d'échecs? Elles nous montrent principalement que la pièce de Middleton, dont les relations étroites avec un épisode précis de l'histoire d'Angleterre sont souvent présentées comme la principale caractéristique et la principale faiblesse, parce qu'elles rendent la pièce incompréhensible ou inintéressante hors de son contexte, appartient en fait, en tant qu'allégorie en forme de jeu d'échecs, à une tradition à la fois ancienne et universelle. Elle s'apparente en particulier aux grandes œuvres humanistes de la Renaissance, où l'allégorie traditionnelle, morale, didactique, édifiante, est utilisée comme véhicule du raffinement, de l'exploration des sens et du savoir par Colonna ou Alberti, comme cadre majestueux de la passion des dieux dans l'épopée tourmentée de Vida, comme spectacle enchanteur offert à la réflexion d'un groupe de fins observateurs parcourant le monde dans l'œuvre de Rabelais.

Le message, les significations multiples de l'allégorie, ne sont bien sûr pas occultées, mais celle-ci acquiert une nouvelle dimension spectaculaire, esthétique et

\footnotetext{
${ }^{46}$ Rabelais, op. cit., chapitre XXIV, p. 1415.
} 
dynamique, qui la rend plus attrayante dans les ouvrages cités que dans des versions antérieures plus austères. Même s'il est impossible de prouver l'influence directe de telle ou telle œuvre sur la pièce de Middleton, les échos relevés matérialisent en quelque sorte une intangible culture européenne moderne, faite d'emprunts au passé agencés selon de nouveaux principes, en réponse à de nouvelles interrogations.

Pour compléter l'évocation de ces échos allégoriques présents dans la Partie d'échecs, il reste à mentionner ses relations avec une tradition anglaise plus archaïque où la métaphore échiquéenne est principalement utilisée pour délivrer un message moral de nature eschatologique, dont l'impact est sans doute considérablement affaibli en $1624^{47}$.

Pour Jane Sherman, qui étudie les sources de l'allégorie de Middleton, l'influence des Moralités et des formes dramatiques de la période Tudor ne doit pas être sous-estimée. Dans sa Partie d'échecs, Middleton utilise un langage dramatique est bien connu du public. M. C. Bradbrook note : «Le jeu d'échecs et le jeu de cartes étaient apparemment deux formes particulièrement populaires des Moralités. » Dans les pièces allégoriques représentant les trois états du royaume (clergé, noblesse, tiers état), le jeu est souvent présent, comme dans la traduction de 1475 de l'ouvrage de Jacques de Cessoles, The Game and Playe of the Chesse par William Caxton.

Le rêve décrit dans l'Induction de la Partie d'échecs, l'entrée solennelle et muette des deux Maisons sous les yeux de l'Erreur et de Saint Ignace sont des procédés typiques des Moralités, ou des 'Estates Plays' décrites par Jane Sherman ${ }^{48}$. Les spectateurs reconnaissent ces emprunts et savent les décoder. Ainsi l'émasculation du Pion du Cavalier Blanc mentionnée à l'acte 1 (145), mutilation physique, est aussitôt assimilée à une faute ou une imperfection morale ou politique par le public. Les Moralités associent en effet souvent une faiblesse physique à un défaut moral, et mettent par exemple en scène un John the Commonweal boiteux

\footnotetext{
${ }^{47}$ Voir le poème de John Skelton (1460-1529) Upon a deedmans hed où la mort est une joueuse d'échecs, ou la moralité du XV ${ }^{\text {ème }}$ siècle Destructorium vitiorum, où l'homme engage une partie avec le Diable, cité par P. Yachnin, 1982, pp. 323-324.

${ }^{48}$ Jane Sherman, «The Pawns’ Allegory in Middleton's A Game at Chess », in RES, n 29, 1978, p. 149.
} 
(tout comme Loyola), ou une Vérité couverte de boutons. Selon le même principe analogique, un glouton comme le Gros Fou sera, avant qu'il ne l'avoue lui-même, assimilé à un personnage avide de pouvoir et d'argent.

Malgré ces emprunts à des formes anciennes, Middleton s'efforce de dépoussiérer, son allégorie et l'habille d'une tenue à la mode. Il actualise ses références échiquéennes en se servant d'un manuel d'échecs particulièrement populaire paru en 1614 et réédité dans une version augmentée en 1618, The Famous Game of Chesse-Playe d'Arthur Saul. L'avertissement adressé à son «Courtois Lecteur », à la fois familier, rassurant et très pédagogique, partage ses vertus avec le Prologue de la pièce, mais aussi ses défauts : tous deux mettent fin prématurément à la curiosité du lecteur, ou du spectateur, en annonçant d'emblée toutes les phases du jeu :

Car dans ce livre tu trouveras d'abord les noms et les formes des pièces, comment les disposer, puis leurs pions, et comment on doit les jouer, et après que je t'aie montré certaines raisons pour lesquelles ce jeu ne saurait avoir de règles (certains prétendent qu'il y en a), alors tu découvriras de nombreuses et plaisantes parties qui t'enchanteront et t'encourageront à t'exercer au jeu : tu apprendras aussi directement ce qu'est un mat aveugle, ce que signifie pat et comment on y peut y parvenir. Enfin, après quelques avertissements sur la façon dont ut dois te comporter en jouant à ce jeu, tu seras informé des lois qui le gouvernent, pour que tu le comprennes correctement et que tu ne souffres d'aucune controverse. Salut, A. S. ${ }^{49}$.

Arthur Saul annonce donc franchement la couleur : le jeu est un divertissement pour lequel il n'y a pas de règles. Cependant, l'auteur s'exécute et décrit, en vers très élégants, le déplacement de chaque pièce :

Tel un chevalier, le Cavalier vient toujours en aide à son Roi,

\footnotetext{
${ }^{49}$ Arthur Saul, The Famous Game of Chesse-Play, 'Being a princely exercise; wherein the Learner may profite more by reading of this small Booke, then by playing of a thousand Mates. ', 'To the Courteous Reader', Londres, 1672 [1618]. L'exemplaire consulté est celui (non paginé) de l'Université d'Illinois, consultable sur EEBO (Early English Books, 1641-1700 / 1468:15). Ma traduction.
} 
Et il saute par dessus les pions et les pièces, brandissant son honneur ${ }^{50}$.

Plus loin, il évoque l'échec à la découverte dans un passage qui, comme l'a observé pour la première fois J. R. Moore, a fortement inspiré Middleton ${ }^{51}$ : « un échec à la découverte, étant l'une des manœuvres les plus élégantes et des plus destructrices que tu puisses infliger à ton adversaire aux échecs ${ }^{52}$. » Il donne ensuite de nombreux exemples de ce coup redoutable, avant de conclure : «Une découverte peut arriver par de nombreux autres chemins, et aboutit souvent à un échec et mat, qui est le plus noble des mats ${ }^{53}$.' Enfin, il précise dans une annexe sur « La diversité des mats » que «L'échec à la découverte [est] le mat le plus difficile à construire. »

J. R. Moore, après avoir trouvé la trace d'un réel savoir échiquéen chez Middleton, qui s'inspire d'un manuel célèbre de l'époque, fait du dramaturge un amateur éclairé. Il croit reconnaître dans la Partie d'échecs la technique du grand maître jésuite italien (mais de nationalité espagnole) Gioachino Greco (1600-1634), qui séjourne en Angleterre, après une tournée triomphale à travers toute l'Europe, de 1622 à 1624. Middleton, selon Moore, veut jouer « contre les Jésuites avec les mêmes armes que leur propre champion ${ }^{54} »$. J. W. Harper, dans son édition de 1966, analyse le début de l'acte I comme un "gambit à la Reine décliné », c'est-à-dire une manœuvre bien précise utilisée par les joueurs pour ouvrir une partie. Mais les limites d'une transposition fidèle des règles du jeu sur la scène apparaissent bien vite. Il n'y a pas dans la pièce les trente-deux acteurs indispensables au déroulement d'une partie en bonne et due forme; l'alternance des coups blancs et noirs est très vite interrompue par l'arrivée du Pion du Fou Noir et sa conversation avec le Pion de la Reine Noire, équivalent scénique d'un impossible coup double sur l'échiquier. Les remarques du Cavalier Noir ne font que confirmer l'abîme qui sépare les règles de la

\footnotetext{
${ }^{50}$ Arthur Saul, op. cit., 'To the Reader'.

${ }^{51}$ Voir J. R. Moore, 'The Contemporary Significance of Middleton's A Game at Chess', in PMLA, n ${ }^{\circ}$ 50, 1935, pp. 761-768.

${ }_{53}^{52}$ Arthur Saul, op. cit., chapitre XVII, 'Of a Discoverie'.

${ }^{53} \mathrm{Ibid}$.

${ }^{54}$ J. R. Moore, op. cit., p. 762.
} 
Partie d'échecs de celles du jeu d'échecs. Au Roi Noir qui voudrait capturer la Reine Blanche, il répond:

Vous êtes trop empressé, Sire.

Si elle était prise, la partie serait terminée.

Mon but est ce Cavalier Blanc ; s'il est piégé d'abord

Le Duc suivra bientôt.

(3.1.244-247)

Une telle analyse ne correspond à aucune stratégie connue, mais s'inscrit dans l'exploitation dramaturgique que fait Middleton des règles du jeu d'échecs. En d'autres termes, la cohérence allégorique est sacrifiée pour que l'allégorie puisse coller au mieux au contenu politique de la pièce. De même, l'échec à la découverte, pourtant décrit précisément par Saul, dont la cause directe devrait être la présence du Duc Blanc derrière son Cavalier, est transformé en acte de bravoure isolé de ce dernier, en triomphe du Cavalier Blanc. Enfin, que dire d'une partie d'échecs où les Pions semblent détenir un pouvoir nettement supérieur à celui des Rois et des Reines, réduits à des rôles proches de la figuration et caractérisés par un manque évident de perspicacité s5 $^{55}$

La partie jouée par ces Rois, Cavaliers et Ducs n'obéit donc pas aux règles strictes du jeu d'échecs, Middleton refusant, comme Saint Ignace, de les respecter, et devient un spectacle total et débridé, une série de pauses et de rebondissements, de tableaux sans cesse changeants, qui s'enchaînent dans l'allégresse, pour faire naitre cette 'facetious comedy' évoquée par John Holles en août 1624. Car la Partie d'échecs n'est pas une simple curiosité dramatique, une sorte de monstre né d'un empilage complexe de blocs empruntés à d'autres œuvres, à d'autres traditions et à d'autres époques, dont le succès ne tient qu'à sa peinture de l'actualité. La pièce de

\footnotetext{
${ }^{55}$ Le Roi Blanc condamne à tort le Pion de la Reine Blanche, la Reine Blanche manque de tomber entre les mains du Gros Fou, le Roi Noir est un impatient qui veut de suite prendre la Reine, la Reine Noire n'intervient que dans dix petits vers...
} 
Middleton est manifestement l'héritière d'un «avant» particulièrement riche, une remarquable satire politique et morale, mais elle n'en est pas moins un exemplaire unique de divertissement parfaitement adapté à son temps ${ }^{56}$, où la pesanteur et la rigidité que pouvaient faire craindre sa lourde hérédité et son enveloppe allégorique omniprésente, s'effacent devant une remarquable légèreté. Le rythme dominant, sans doute insufflé par l'imitation des mouvements du jeu et son alternance entre Noirs et Blancs, ses changements de direction, ses revirements, est celui des city comedies, où toute une troupe de personnages pittoresques évolue dans les rues et les échoppes de Londres dans un rythme souvent endiablé.

Les seules représentations professionnelles de la pièce datent de 1624. Les compagnies étudiantes d'Oxford et de Cambridge présentent la pièce en 1971 et 1973 respectivement, et rencontrent un franc succès. Pour le critique du Times, «le spectacle est tellement plaisant que l'on se demande pourquoi les compagnies professionnelles ont laissé la pièce prendre la poussière pendant si longtemps ${ }^{57}$ », et Mark Lambirth, le metteur en scène de l'adaptation présentée par la Queen's College Dramatic Society de Cambridge en 1973, indique que «le public s'est bien amusé $^{58} \gg$.

Par un heureux hasard du calendrier, la pièce a été sélectionnée par l'atelier Read not Dead (Lues et pas mortes) du théâtre du Globe, dont le but est de faire (re)découvrir des pièces peu connues ou rarement jouées lors de séances de travail ouvertes au public. Une nouvelle Partie d'échecs sera donc à nouveau jouée le 2 décembre 2018, à l'endroit même où elle a vu le jour près de 400 ans auparavant.

\section{Liste des ouvrages cités}

N. W. Bawcutt, « New Light on Middleton's Knowledge of Chess. », Notes and Queries, $\mathrm{n}^{\circ} 34,1987$, pp. 301-2.

Michael Billington, «A Game at Chess », in The Times, 29 mai 1971.

\footnotetext{
${ }^{56}$ Rappelons que le théâtre est à l'époque un divertissement très populaire qui touche toutes les classes sociales.

${ }^{57}$ Critique de Michael Billington, parue dans The Times, 29 Mai 1971. Ma traduction.

${ }^{58}$ Mark Lambirth, metteur en scène. Lettre à l'auteur du 4 juin 1999. Ma traduction.
} 
M. C. Bradbrook, English Dramatic Form: A History of its Development, Londres, 1965.

Jacques de Cessoles, Game and Play of the Chesse, trad. William Caxton, éd. William E. A. Axon. Londres, 1883.

George Chapman, Bussy d'Ambois, éd. Nicholas Brooke, The Revels Plays, Londres, Methuen, 1964.

Swapan Chakravorty, Society and Politics in the Plays of Thomas Middleton, Oxford, Clarendon Press, 1996.

Clare, Janet, 'Art Made Tongue-Tied by Authority' : Elizabethan and Jacobean Dramatic Censorship, Manchester, Manchester University Press, 1990.

T. S . Eliot, Elizabethan Essays, Londres, Faber, 1934.

Antoine Ertlé, éd. Thomas Middleton, A Game at Chess/ Une Partie d'échecs : édition bilingue établie par Antoine Ertlé, Paris, Epistémè, Université de la Sorbonne Nouvelle $\quad-\quad$ Paris $\quad 3, \quad 2004 . \quad$ (http://www.etudesepisteme.org/ee/articles.php?lng=fr\&pg=54).

Dorothy M. Farr, Thomas Middleton and the Drama of Realism: A Study of some Representative Plays, Edinburgh, Oliver \& Boyd, 1973.

David F. George, «Thomas Middleton's Sources: A Survey», $N \& Q, \mathrm{n}^{\circ} 216$, 1971, p. 17-22.

T. H. Howard-Hill, «The Origins of Middleton's A Game at Chess. », in Research Opportunities in Renaissance Drama, $\mathrm{n}^{\circ} 28,1985$, pp. 3-14.

---. «Political Interpretations of Middleton's A Game at Chess (1624).», in Yearbook of English Studies, ${ }^{\circ} 21,1991$, pp. 274-85.

---. éd. A Game at Chess, de Thomas Middleton, The Revels Plays, Manchester, Manchester University Press, 1993.

---. Middleton's "Vulgar Pasquin". Essays on A Game at Chess, Newark, University of Delaware Press, 1995.

Emanuela Kretzulesco-Quaranta, Les jardins du songe, Paris, 1986. 
John Lavagnino et Gary Taylor (dir.), Thomas Middleton and Early Modern Textual Culture: A Companion to the Collected Works, Oxford, Oxford University Press, 2007.

Liane Lefaivre, Leon Battista Alberti's Hyptnerotomachia Poliphili, Boston, Mass., 1997.

Pierre Laurens et C. Balavoine, Musae Reduces, Anthologie de poésie latine européenne de la Renaissance, Leiden, 1975.

John Robert Moore, «The Contemporary Significance of Middleton's Game at Chess. », in $P M L A, \mathrm{n}^{\circ}$ 50, 1935, pp. 761-68.

H. J. R. Murray, A History of Chess, Oxford, Clarendon Press, 1913.

Jean-Michel Péchiné, Les échecs. Roi des jeux, jeu des rois, Découvertes Gallimard, Paris, Gallimard, 1997.

François Rabelais, Gargantua et Pantagruel, éds. Jean Céard, Gérard Defaux \& Michel Simonin, Paris, Librairie Générale Française, 1994.

---. Euvres complètes, éds. Mireille Huchon \& François Moreau, Bibliothèque de la Pléiade, Paris, Gallimard, 1994.

Gary Taylor et John Lavagnino (dir.), Thomas Middleton: The Collected Works, Oxford, Oxford University Press, 2007.

Arthur Saul, The Famous Game of Chess Play, édition révisée par Jean Barbier, Londres, 1672 [1618]. EEBO (Early English Books, 1641-1700 / 1468:15).

J. Shami, «Thomas Middleton's A Game at Chess: A Sermon Analogue. », in Notes and Queries, $\mathrm{n}^{\circ} 240,1995$, pp. 367-69.

Jane Sherman, «The Pawns' Allegory in Middleton's A Game at Chess. », in Review of English Studies, ${ }^{\circ} 29,1978$, pp. 147-59.

Richard Stanton Lambert, éd. \& trad. The Game at Chess (Scacchia Ludus), de Marco Girolamo Vida, Londres, The Stanton Press, 1921.

Paul Yachnin, «A Game at Chess and Chess Allegory. », in Studies in English Literature, 1500-1900, n 22, 1982, pp. 316-30. 
---. «A Game at Chess: Thomas Middleton's Praise of Folly.», in Modern Language Quarterly, ${ }^{\circ}$ 48, 1987, pp. 107-23.

---. Stage-Wrights: Shakespeare, Jonson, Middleton, and the Making of Theatrical Value, Philadelphie, University of Philadelphia Press, 1997.

\section{Annexe}

Identité des pièces de l'allégorie

\begin{tabular}{|c|c|}
\hline $\begin{array}{l}\text { Roi Blanc }=\text { Jacques } I^{\text {er }} \\
\text { d'Angleterre }\end{array}$ & Roi Noir = Philippe IV d'Espagne \\
\hline $\begin{array}{l}\text { Reine Blanche = Incarnation de } \\
\text { l'Église d'Angleterre }\end{array}$ & $\begin{array}{l}\text { Reine Noire = Reine d'Espagne } \\
\text { (ou l'Infante Maria, ou l'Église de } \\
\text { Rome) }\end{array}$ \\
\hline Cavalier Blanc $=$ Prince Charles & $\begin{array}{l}\text { Cavalier Noir }=\text { Comte de } \\
\text { Gondomar }\end{array}$ \\
\hline Duc Blanc = Duc de Buckingham & Duc Noir $=$ Comte-duc d'Olivares \\
\hline $\begin{array}{l}\text { Fou Blanc = George Abbott, } \\
\text { archevêque de Canterbury }\end{array}$ & $\begin{array}{l}\text { Fou Noir = Père Général des } \\
\text { Jésuites }\end{array}$ \\
\hline $\begin{array}{l}\text { Pion du Roi Blanc }=\text { Comte de } \\
\text { Middlesex }\end{array}$ & Pion du Fou Noir $=$ un Jésuite \\
\hline $\begin{array}{l}\text { Pion de la Reine Blanche }= \\
\text { Incarnation de la dévotion, de la } \\
\text { foi protestante }\end{array}$ & $\begin{array}{l}\text { Pion de la Reine Noire = une } \\
\text { Jésuitesse }\end{array}$ \\
\hline $\begin{array}{l}\text { Gros Fou = Archevêque de } \\
\text { Spalato }\end{array}$ & $\begin{array}{l}\text { Gros Fou = Archevêque de } \\
\text { Spalato }\end{array}$ \\
\hline
\end{tabular}

Antoine Ertlé, Université Bordeaux Montaigne 